\title{
Theoretical and experimental analysis of the equilibrium contours of liquid bridges of arbitrary shape
}

\author{
J. M. Montanero, G. Cabezas, and J. Acero \\ Departamento de Electrónica e Ingenieria Electrontecanica. Universidad de Extrenadura, \\ E-06071 Badajoz, Spain \\ J. M. Perales \\ E.T.S.I. Aeronatuicos, Universidad Politecnica de Madrid, E-28040 Madrid, Spain
}

\begin{abstract}
The equilibrium shape of the liquid bridge interface is analyzed theoretically and experimentally. Both axisymmetric and nonaxisymmetric perturbations are considered. The axisymmetric deviations are those related to volume effects, the difference between the radii of the disks, and the axial forces acting on the liquid bridge. The nonaxisymmetric deviations are those due to the eccentricity of the disk and the action of lateral forces. The theoretical study is performed using three different teclnniques: (i) an analytical expansion around the cylindrical solution. (ii) a finite difference scheme, and (iii) an approximate numerical approach valid only for slight nonaxisymmetric deviations. The results of the three methods are compared systematically. There is a very good agreement between the analytical and the numerical approaches for contours which are close to cylindrical, and the agreement extends to configurations with only moderate deviations from cylindrical. Experiments are performed using the so-called neutral buoyancy or plateau technique. Theoretical and experimental contours are compared considering a wide range of values for the parameters characterizing the perturbations. In general, the finite difference method provides reasonably accurate predictions even for large deviations of the liquid bridge contour from cylindrical.
\end{abstract}

\section{INTRODUCTION}

The mechanical behavior of liquid bridges has been a frequent subject of analysis over the last few decades. Apart from their basic scientific interest. liquid bridges show undoubted technological potential. The liquid bridge model has been used to analyze such different phenomena as melt-zone refining, 'flow in porous media or dispersions as in coating processes. ${ }^{2}$ As far as the static problem is concerned. studies have focused on the calculation of both the liquid bridge equilibrium interface shape $e^{3,4}$ and the stability limit. ${ }^{3-6}$ In the liquid bridge problem, the axial (lateral) Bond number indicates the relative magnitude of the surface tension forces and of the axial (lateral) force acting on the liquid bridge. The values of the Bond numbers can be inferred from the deformation of the liquid bridge contour. This can be done by fitting theoretical predictions to experimental equilibrium shapes. In this way, liquid bridges can be used either as fluid accelerometers or as tensiometers. In the former case, the goal is to measure small accelerations under microgravity conditions. ${ }^{7,8}$ In the latter case. the liquid bridge is used to obtain the value of the surface tension associated with the interface between two immiscible liquids of similar densities. Obviously. for these purposes it is essential to be able to accurately predict the liquid bridge interface shape as a function of the parameters characterizing the problem.

The calculation of the equilibrium interface shape can also be considered as a necessary step in the theoretical analysis of the liquid bridge dynamics under certain conditions. For instance, the interface curvature has a pronounced influence on the stability of the oscillatory thermocapillary convection in liquid bridges. ${ }^{9.10}$ In most of the theoretical studies of this flow, the interface position is assumed unperturbed by flow disturbances. Therefore, the calculation of the interface shape used in these studies is a particular case of the problem dealt with in the present paper.

To obtain the liquid bridge contour one must integrate the (exact) Young-Laplace equation. which represents the dynamical equilibrium between pressure. surface tension. gravity and inertial forces. This integration has to be performed taking into account certain boundary conditions as well as the continuity equation. Most studies have dealt with axisymmetric configurations for which the mathematics is much simpler. In this case the capillary equation becomes a nonlinear ordinary differential equation that can be numerically integrated using the standard Runge-Kutta method. This is a typical two-point boundary value problem that can be solved by finding the corresponding initial conditions. An additional constraint is that the volume defined by the contour has to coincide with the liquid bridge volume. This numerical problem has been successfully solved and the axisymmetric liquid bridge contour can be obtained with any desired accuracy. ${ }^{8}$ If we consider either lateral inertial forces or a displacement between the centers of the supporting disks, the liquid bridge contour becomes nonaxisysmmetric. In this case, the Young-Laplace equation becomes a nonlin- 
ear partial differential equation, and its integration preserving the continuity condition is a complicated problem.

There would be obvious advantages in obtaining analytical expressions for the liquid bride equilibrium interface shape. For instance, analytical expressions have been used to easily fit theoretical equilibrium contours to experimental ones, so that the liquid bridge can be used as a fluid accelerometer. ${ }^{7,8}$ Also. a finite difference scheme has been used to numerically obtain the liquid bridge contour, and the results obtained for the stability limits were in good agreement with those calculated using asymptotic techniques around the cylinder., ${ }^{3,4}$ The stability of the equilibrium configurations has also been investigated using another numerical procedure which involved solving for energy-minimizing surface configurations. ${ }^{611}$ The results were found to be in agreement with the experimental data. ${ }^{6}$ In principle, there are no restrictions on the finite difference method although convergence is not guaranteed. To the best of our knowledge, a detailed comparison of theoretical and experimental contours considering both axisymmetric and nonaxisymmetric perturbations has not yet been carried out.

To check the validity of the theoretical predictions, we performed a comparison with experiment. In order to obtain stable fluid configurations with large sizes, one has to perform the experiments under microgravity conditions. The expense and difficulty involved in performing space and inflight experiments have forced researchers to develop experimental support on Earth. In the neutral buoyancy technique (the so-called Plateat-tank simulation), the liquid bridge is surrounded by an outer liquid with similar density to partially compensate for the effect of the hydrostatic pressure along the interface. This technique has often been used to simulate microgravity conditions. ${ }^{6}$

The organization of the paper is as follows. The mathematical problem is formulated in Sec. II. The formalism of the expansion used to analytically calculate the liquid bridge interface shape is presented in Sec. III. In Sec. IV we describe the numerical methods. A comparison between the different theoretical approaches is presented in Sec. V. The experimental procedure and the results obtained are described in Sec. VI. Finally, in Sec. VII we present some concluding remarks.

\section{MATHEMATICAL MODEL}

The fluid configuration considered is sketched in Fig. 1 It consists of an isothermal mass of liquid of volume $v$, held between two parallel disks placed a distance $L$ apart. The radii of the disks are $R_{1}=R_{0}(\mathrm{I}-H)$ and $R_{2}=R_{0}(1+H) . R_{0}$ being the mean radius used as the characteristic length. In general. the disks are not coaxial. $2 E$ being taken as the displacement distance between the axes of the disks. The liquid bridge is subjected to the action of both axial and lateral constant forces, $g_{a}$ and $g_{l}$ being their corresponding magnitudes per unit mass. The angle between the displacement direction of the disk axes (the $r$ axis) and the direction of the lateral force is $\alpha$. The liquid bridge is surrounded by another fluid medium (either a gas or another liquid), $\Delta p$ being the difference between the liquid bridge and the sur-

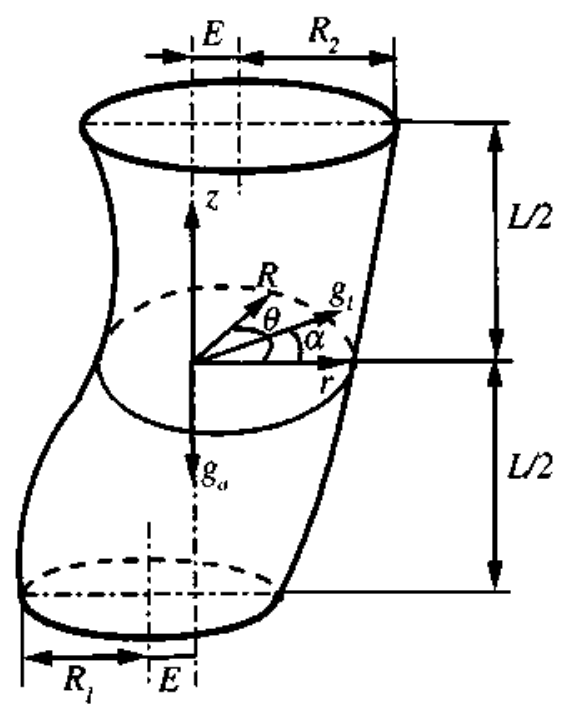

FIG. 1. Geometry and coordinate system for the fluid configuration considered.

rounding medium densities. The surface tension associated with the interface is $\sigma$. The contour of the liquid bridge is characterized by the function $R(z, \theta)$, which measures the distance between a surface element and the $z$ axis

The set of dimensionless parameters $\left\{V, \Lambda, H, B_{a}\right.$. $\left.B_{I}, e, \alpha\right\}$ uniquely characterizes the above fluid configuration. Here, $V \equiv \mathcal{V} /\left(\pi R_{0}^{2} L\right)$ is the reduced volume defined as the ratio of the physical volume $\mathcal{V}$ to the volume of a cylinder of the same length $L$ and radius equal to $R_{0}$. The parameter $\Lambda \equiv L /\left(2 R_{0}\right)$ is the slenderness. Axisymmetric perturbations are represented by the geometrical parameter $H$ and the axial Bond number $B_{a} \equiv \Delta \rho g_{a} R_{j}^{2} / \sigma$. Nonaxisymmetric perturbations are represented by the lateral Bond number $B_{l}$ $\equiv \Delta \rho g_{i} R_{0}^{2} / \sigma$, the dimensionless eccentricity $e \equiv E / R_{0}$, and the angle $\alpha$

The equilibrium interface shape of the liquid bridge is described by the (exact) capillary equation

$$
C(z, \theta)-B_{c} z+\frac{1}{2} W R^{2}+B_{l} R \cos (\theta-\alpha)+P=0 .
$$

where $C(z, \theta)$ is twice the (dimensionless) local mean curvature at a point on the interface

$$
\begin{aligned}
C(z, \theta)= & {\left[R\left(1+R_{z}^{2}\right)\left(R_{\theta \theta}-R\right)+R R_{z z}\left(R^{2}+R_{\theta}^{2}\right)\right.} \\
& \left.-2 R_{\theta}\left(R_{\theta}+R R_{z} R_{z \theta}\right)\right]\left[R^{2}\left(1+R_{z}^{2}\right)+R_{\theta}^{2}\right]^{-3 / 2} .
\end{aligned}
$$

In the above expressions, the coordinate $z$ and the function $R$ have been made dimensionless with the characteristic length $R_{0}$. The subscripts $z$ and $\theta$ indicate derivatives with respect to $z$ and $\theta$, respectively. The capillary pressure $P$ $\equiv \Delta p_{0} R_{0} / \sigma$ is a dimensionless constant which is related to the difference $\Delta p_{0}$ between the outer pressure and the inner pressure at the origin of the coordinate system. In addition, the centrifugal force term $1 / 2 W R^{2}$ ( $W$ is the Weber number) has been introduced. The motivation will be discussed in the next paragraph. Equilibrium interface shapes are calculated by integration of the partial differential equation (1a) with the boundary conditions 


$$
\begin{aligned}
& R( \pm \Lambda, \theta)=\left[(\mathbf{1} \pm H)^{2}-e^{2} \sin ^{2} \theta\right]^{1 / 2} \pm e \cos \theta . \\
& R(z, \theta+2 \pi)=R(z, \theta) .
\end{aligned}
$$

The value of the capillary pressure $P$ is unknown and must be indirectly determined by imposing conservation of the volume, i.e..

$$
\int_{-\Lambda}^{\Lambda} d z \int_{0}^{2 \pi} d \theta R^{2}=4 \pi \Lambda \mathrm{Y}^{2}
$$

The expressions (1) define the mathematical problem dealt with in the present paper. The inclusion of the Weber number as an additional parameter is unusual but relevant. In fact. the eccentric rotation of the liquid bridge regarded as a solid body in the absence of lateral forces acting on it ${ }^{12}$ is also formally described by (1). Let $\omega$ and $\widetilde{E}$ be the constant angular speed and the distance of the rotation axis from the $z$ axis, respectively. The capillary equation becomes

$$
C(z, \theta)-B_{a} z+\frac{1}{2} W\left[R^{2}-2 \widetilde{e} R \cos (\theta+\widetilde{\alpha})\right]+P=0,
$$

where $W \equiv \Delta \rho \omega^{2} R_{0}^{3} / \sigma$ is the Weber number, $\tilde{e} \equiv \tilde{E} / R_{0}$. and $\tilde{\alpha}$ is the angle formed by the $r$ axis and the displacement direction of the rotation axis with respect to the $z$ axis. It is easy to verify that, if we set $B_{l}=W \tilde{e}$ and $\alpha=\pi-\tilde{\alpha}, \mathrm{Eq}$. (1a) becomes Eq. (2). The boundary conditions (1c) and (1d) as well as the continuity equation (le) are the same for both problems. Therefore, the mathematical model (1) also describes the eccentric rotation of the liquid bridge regarded as a solid body.

\section{ANALYTICAL SOLUTION}

The fluid configuration described above is, in general. subjected to the action of both axisymmetric and nonaxisymmetric perturbations. If the parameters characterizing these perturbations vanish ( $V=1$ and $B_{a}=H=W=B_{I}=e$ $=0$ ), the solution of problem (I) is cylindrical $(R=P=\mathbf{I})$ independently of the slenderness $\Lambda$. In this section we calculate the equilibrium interface shape assuming that the deviations from the cylinder are sufficiently small. Under this condition the liquid bridge is assumed to be stable. This hypothesis in fact holds for configurations close to cylindrical and slenderness less than $\pi$ (Plateau-Rayleigh stability limit). ${ }^{5}$ In general, the bifurcation theory methods can be used to rigorously establish the existence of equilibrium solutions close to known ones. ${ }^{13}$ Let us expand the expressions for the liquid bridge interface shape $R(z, \theta)$ and for the capillary pressure $P$ in powers of the perturbation parameters $\epsilon_{1} \equiv B_{a} . \epsilon_{2} \equiv V-1 . \epsilon_{3} \equiv H . \epsilon_{4} \equiv W, \epsilon_{5} \equiv e, \epsilon_{6} \equiv B_{l}$

$$
\begin{aligned}
& R(z, \theta)=\mathbf{I}+\sum_{i} \epsilon_{i} r_{i}(z, \theta)+\sum_{i, j} \epsilon_{i} \epsilon_{j} r_{i j}(z, \theta)+\cdots \\
& P=1+\sum_{i} \epsilon_{i} p_{i}+\sum_{i, j} \epsilon_{i} \epsilon_{j} p_{i j}+\cdots
\end{aligned}
$$

Since the convergence of the power series (3) is not guaranteed, they should be considered in principle as a formal solution. One has to determine the dependence of the functions $r_{i}$ and $r_{i j}$ on the $z$ and $\theta$ coordinates, as well as the param- eters $p_{i}$ and $p_{i j}$. Inserting the expansions (3) into Eq. (1), and retaining terms of order $\epsilon_{i}$, one easily finds

$$
\begin{aligned}
& r_{i_{z z}}+r_{i \partial \theta}+r_{i}+p_{i}-\delta_{i 1} z+\frac{1}{2} \delta_{i 4}+\delta_{i 6} \cos (\theta-\alpha)=0, \\
& r_{i}( \pm \Lambda, \theta)= \pm \delta_{i 3} \pm \delta_{i 5} \cos \theta \\
& r_{i}(z, \theta+2 \pi)=r_{i}(z, \theta) \\
& \int_{-\Lambda}^{\Lambda} d z \int_{0}^{2 \pi} d \theta r_{i}=2 \pi \Lambda \delta_{i 2} .
\end{aligned}
$$

In the above equations. $\delta_{i j}$ denotes the Kronecker symbol. The solution of the set of linear equations (4) is given in the Appendix. The functions $r_{2}(z)$ and $r_{6}(z, \theta)$ correspond to symmetric deformations with respect to the middle plane parallel to the disks $z=0$, whereas $r_{1}(z), r_{3}(z)$, and $r_{5}(z, \theta)$ are antisymmetric. The former imply a C-type deformation with respect to the cylinder and the later an amphora-type perturbation.

The order $\epsilon_{i} \epsilon_{j}$ leads to the following set of equations:

$$
\begin{aligned}
& r_{i j}+r_{i j \theta \theta}+r_{i j}+p_{i j}-r_{i j} r_{j}+\frac{1}{2} r_{i z} r_{j z}-\frac{1}{2} r_{i \theta} r_{j \theta}-r_{i} r_{j \theta \theta} \\
& \quad-r_{i_{\theta \theta}} r_{J}+\left(1+\delta_{i 4}\right) \delta_{j 4} r_{1}+\frac{1}{2\left(1+\delta_{i j}\right)}\left[\left(1+\delta_{i \sigma}\right)\right. \\
& \left.\quad \times \delta_{j 6} r_{i}+\left(1+\delta_{j 6}\right) \delta_{i 6} r_{j}\right] \cos (\theta-\alpha)=0, \\
& r_{i j}( \pm \Lambda, \theta)=-\frac{1}{2} \delta_{i 5} \delta_{j 5} \sin ^{2} \theta \\
& r_{i j}(z, \theta+2 \pi)=r_{i j}(z . \theta), \\
& \int_{-\Lambda}^{\Lambda} d z \int_{0}^{2 \pi} d \theta\left(2 r_{i j}+r_{i j} r_{j}\right)=0 .
\end{aligned}
$$

The solution of the second-order problem defined by (5) is also given explicitly in the Appendix. It must be pointed out that this calculation has already been performed for $W=0{ }^{7}$ Nevertheless. due to several misprints in the expressions presented in the Appendix of Ref. 7, the results do not coincide for several functions. The solution $r_{i_{j}}$ is symmetric if $i+j$ is even. and antisymmetric otherwise. Also. the effects associated with the eccentricity and the lateral force acting on the liquid bridge imply symmetric deformations with respect to the planes $\theta=0$ and $\theta=\alpha$, respectively. Therefore. $r_{i 5}(z, \theta)$ $(i=1.2, \ldots, 5)$ is symmetric with respect to $\theta=0$. while $r_{i 6}(z, \theta)(i=1,2, \ldots, 6 i \neq 5)$ is symmetric with respect to $\theta$ $=\alpha$. Finally, the function $r_{56}(z, \theta)$ is symmetric with respect to $\theta=\alpha / 2$. If the displacement direction of the axes of the disks and the direction of the lateral force coincide ( $\alpha$ $=0$ ), $\theta=0$ constitutes a plane of symmetry. This statement also holds for the full solution $R(z, \theta)$.

\section{NUMERICAL APPROACHES}

\section{A. Finite difference scheme}

Because of the large number of parameters involved and the complicated mathematical structure of the problem (1), the search for exact analytical solutions is impractical. For this reason, numerical techniques constitute a useful alternative. We here present a finite difference scheme. The procedure is similar to that described in Refs. 3 and 4 . Let us 


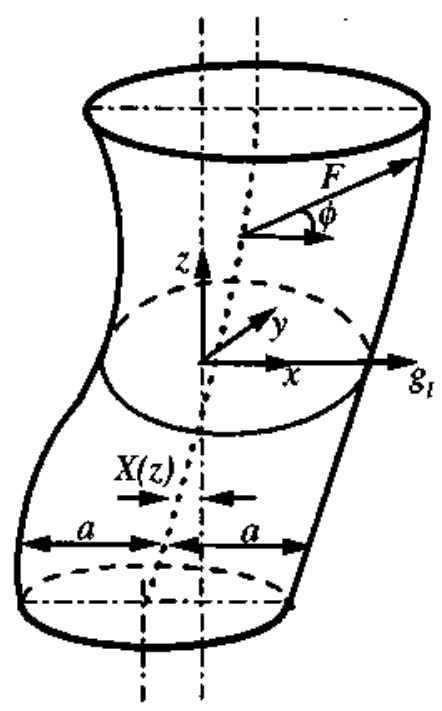

FIG. 2. Geometry and coordinate systen used in the finite difference scheme.

introduce the coordinate system shown in Fig. 2. The origin for each section is located on the midline (dotted line in Fig 2 ). which is equidistant from the interface along the $x$ and $y$ axes. The position of the midline is given by the auxiliary functions $Y(z)$ and $Y(z)$. In what follows we consider the particular case $\alpha=0$. Therefore, $\theta=0(\phi=0)$ constitutes a plane of symmetry and so $Y(z)=0$. The liquid bridge contour is characterized by the function $F(z, \phi)$ which is the distance of a surface element from the midline. The YoungLaplace equation (1a) becomes

$$
\begin{gathered}
M(z, \phi)-B_{a} z+\frac{1}{2} W\left(F^{2}+2 Y F \cos \phi+X^{2}\right) \\
+B_{f}(X+F \cos \phi)+P=0 .
\end{gathered}
$$

where

$$
\begin{aligned}
M(z, \phi) & \\
= & \left\{\left[1+\left(X_{z}+F_{z} \cos \phi\right)^{2}+F_{z}^{2} \sin ^{2} \phi\right]\right. \\
& \times\left(F^{2}+2 F_{\phi}^{2}-F F_{\phi \phi}\right)+\left(F^{2}+F_{\phi}^{2}\right) \\
& \times\left[-X_{z}\left(F_{\phi} \sin \phi+F \cos \phi\right)-F_{z z}\right] \\
& \left.-2\left(F_{z} F_{\phi}-F F_{z \phi}\right)\left[F_{z} F_{\phi}+Y_{z}\left(F_{\phi} \cos \phi-F \sin \phi\right)\right]\right\} \\
& \times\left\{\left[1+\left(X_{z}+F_{z} \cos \phi\right)^{2}+F_{z}^{2} \sin ^{2} \phi\right]\right. \\
& \left.\times\left(F^{2}+F_{\phi}^{2}\right)-\left[X_{z}\left(F_{\phi} \cos \phi-F \sin \phi\right)+F_{z} F_{\phi}\right]^{2}\right\}^{-3 / 2} .
\end{aligned}
$$

In the above expressions, the coordinate $z$ and the functions $F$ and $Y$ have been made dimensionless with the characteristic length $R_{0}$. To calculate the functions $F(z, \phi)$ and $Y(z)$, Eq. (6a) has to be integrated with the boundary conditions

$$
F( \pm \Lambda . \phi)=1 \pm H, \quad F(z, \phi)=F(z, \phi+2 \pi),
$$

and with the volume constraint

$$
\int_{-\Lambda}^{\Lambda} d z \int_{0}^{2 \pi} d \phi F^{2}=4 \pi \Lambda V
$$

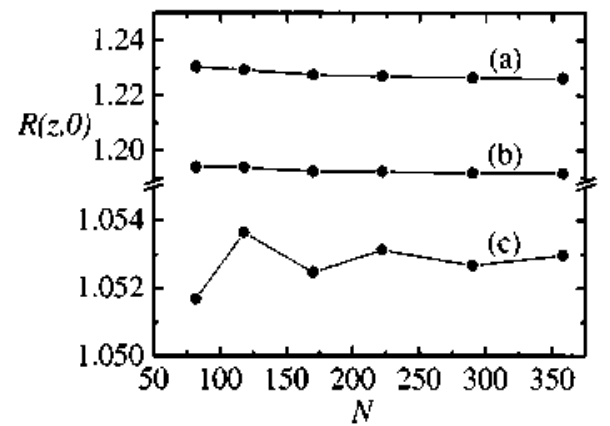

FIG. 3. $R(z, 0)$ for (a) $z=-\Lambda / 2$. (b) $z=0$, and (c) $z=\Lambda / 2$ computed using meshes of different sizes. The values of the parameters characterizing the liquid bridge are: $V=1.1, \Lambda=2, H=0.05, B_{\alpha}=0.15, W=0, B_{t}=0.05$, e $=0, \alpha=0$.

Taking into account the definition of the midline

$$
X( \pm \Lambda)= \pm e, \quad F(z, 0)=F(z, \pi) .
$$

Finally, because $\phi=0$ constitutes a plane of symmetry

$$
F(z, \phi)=F(z, 2 \pi-\phi)
$$

In order to develop a finite difference scheme, the continuum integration domain is replaced by a rectangular mesh with $I \times J$ nodes, $I$ and $J$ being the number of nodes along the $\phi$ and $z$ axes, respectively. The partial derivatives in Eq. (6b) are replaced by central finite differences on the $(I-2) \times(J$ -2 ) inner points of the mesh. Equations (6c) reduce to $2 I$ $+J-2$ conditions for the boundary nodes. The counterpart of integral (6d) is obtained using the extended trapezoidal nule. ${ }^{14}$ The result is a nonlinear relation in which all the nodes are involved. Finally. the symmetry relations (6e) and (6f) lead to $2 J-2$ additional constraints in the corresponding discrete problem.

After the problem represented by (6) has been discretized, the goal is to solve a set of $N=I \times J+1+J$ nonlinear equations to obtain $F$ at the nodes, the capillary pressure $P$, and the position of the midline $X$. In Refs. 3 and 4 this set of equations has been solved for different particular cases once the equations are linearized around a previously obtained solution. We here deal with the nonlinear problem by means of the Newton-Raphson method, ${ }^{14}$ thereby avoiding approximations associated with the linearization. The algorithm is found to be stable. Starting from the cylindrical solution slightly perturbed by a random function, the NewtonRaphson method converges to the sought values of $F . P$. and $X$ after several (less than 15) iterations. In all cases. the liquid bridge equilibrium contour is smooth so that the mesh size may be presumed not to play a relevant role. Figure 3 shows the value $R(z, 0)=F(z, 0)+Y(z)$ for $z=-\Lambda / 2,0$ and $\Lambda / 2$ computed using meshes of different sizes. As can be observed. $R(z, 0)$ becomes practically insensitive to the mesh size for $N>300$. More precisely the differences between the results obtained for $N=290$ and $N=358$ only concern the fourth significant figure. In what follows, we will perform the calculations using a rectangular mesh with $I=16$ and $J$ $=21(N=358)$. In the final step, spline interpolation functions are constructed to provide $F(z, \phi)$ and $Y(z)$ over the continuum integration domain. 

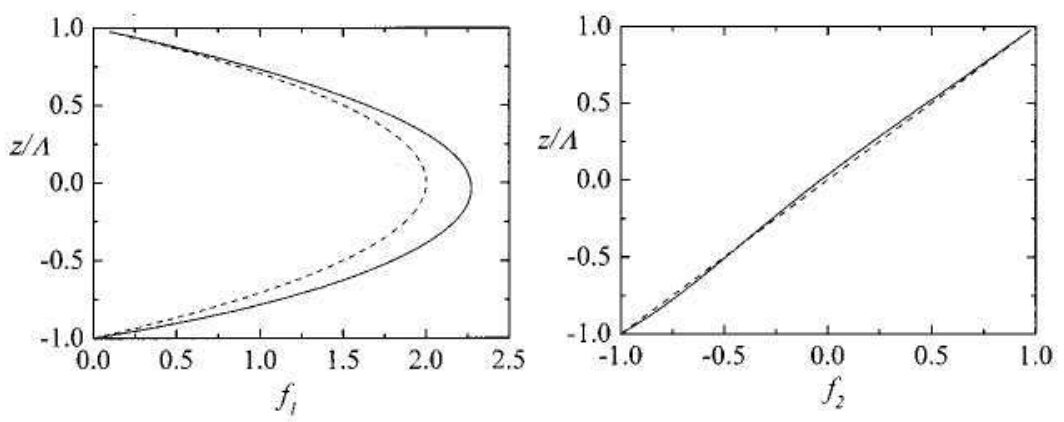

FIG. 4. Plot of the functions $f_{i}(z)$ (solid lines) for $V$ $=1.1, \Lambda=2, H=0.05, B_{a}=0.15$, and $W=0$. The dashed lines correspond to $f_{1}(z)=\frac{1}{2}\left(\Lambda^{2}-z^{2}\right)$ and $f_{2}(z)=z / \Lambda$.
This numerical procedure has been automated and can be easily implemented inside other codes. A Mathematica package, programmed by the authors (the package can be obtained upon request), directly provides the numerical value of the function $R\left(V, \Lambda, H, B_{a}, W, B_{l}, e ; z, \theta\right)$ without other specifications. This is a major advantage, especially when theoretical contours have to be fitted to experimental ones to calculate, for instance, the inertial forces acting on the liquid bridge.

\section{B. Approximate numerical approach}

The calculation of the liquid bridge shape for axisymmetric configurations is much simpler. In this case, the problem can be formulated by the expressions

$$
\begin{aligned}
& \frac{\tilde{R}_{z z}}{\left(1+\widetilde{R}_{z}^{2}\right)^{3 / 2}}-\frac{1}{\widetilde{R}\left(1+\widetilde{R}_{z}^{2}\right)^{1 / 2}}-B_{a} z+\frac{1}{2} W \tilde{R}^{2}+\tilde{P}=0, \\
& \widetilde{R}( \pm \Lambda)=1 \pm H, \\
& \int_{-\Lambda}^{\Lambda} d z \widetilde{R}^{2}=2 \Lambda V,
\end{aligned}
$$

where $\tilde{R}\left(V, \Lambda, H, B_{a}, W ; z\right)$ and $\tilde{P}\left(V, \Lambda, H, B_{a}, W\right)$ are the searched solution. The integration of Eq. (7a) with the boundary conditions ( $7 \mathrm{~b}$ ) is a standard two-point boundaryvalue problem that is solved by transforming it to an initialvalue problem (shooting method). The integration of (7a) is performed using the Runge-Kutta method. The values of $\widetilde{R}_{z}(-\Lambda)$ and $\widetilde{P}$ which lead to $\widetilde{R}(\Lambda)=1+H$ while preserving the volume of the liquid bridge are found by means of a variant of the secant method.
Assume that, given the set of parameters $\left\{V, \Lambda, H, B_{a}, W\right\}$, the corresponding axisymmetric contour $\widetilde{R}(z)$ and the capillary pressure $\widetilde{P}$ are known. We now are interested in the (small) deviations of the equilibrium shape $R(z, \theta)$ from $\widetilde{R}(z)$ due to nonaxisymmetric perturbations. Let us expand the function $R(z, \theta)$ and the capillary pressure $P$ in powers of the small parameters $\widetilde{\epsilon}_{1} \equiv B_{l}$ and $\tilde{\epsilon}_{2} \equiv e$, i.e.,

$$
\begin{aligned}
& R(z, \theta)=\tilde{R}(z)+\tilde{\boldsymbol{\epsilon}}_{1} \widetilde{r}_{1}(z, \theta)+\tilde{\boldsymbol{\epsilon}}_{2} \tilde{r}_{2}(z, \theta)+\cdots, \\
& P=\tilde{P}+\tilde{\boldsymbol{\epsilon}}_{1} \tilde{p}_{1}+\tilde{\boldsymbol{\epsilon}}_{2} \tilde{p}_{2}+\cdots .
\end{aligned}
$$

One now has to determine the dependence of the functions $\tilde{r}_{1}$ and $\tilde{r}_{2}$ on the $z$ and $\theta$ coordinates, as well as the parameters $\tilde{p}_{1}$ and $\tilde{p}_{2}$. Inserting the expansions (8) into Eqs. (1), and retaining terms of order $\tilde{\epsilon}_{i}$, one finds

$$
\begin{gathered}
{\left[1+W \widetilde{R}^{3}\left(1+\widetilde{R}_{z}^{2}\right)^{1 / 2}\right] \widetilde{r}_{i}+\tilde{r}_{i_{\theta \theta}}+\frac{\tilde{R} \widetilde{R}_{z}\left(1+\widetilde{R}_{z}^{2}-3 \tilde{R}_{z z}\right)}{\left(1+\widetilde{R}_{z}^{2}\right)^{2}} \widetilde{r}_{i_{z}}} \\
+\frac{\widetilde{R}^{2}}{1+\widetilde{R}_{z}^{2}} \tilde{r}_{i_{z z}}+\widetilde{R}^{2}\left(1+\widetilde{R}_{z}^{2}\right)^{1 / 2}\left[\delta_{i 1} \widetilde{R} \cos (\theta-\alpha)+\tilde{p}_{i}\right]=0,
\end{gathered}
$$

$\widetilde{r}_{i}( \pm \Lambda, \theta)= \pm \delta_{i 2} \cos \theta$,

$\tilde{r}_{i}(z, \theta+2 \pi)=\tilde{r}_{i}(z, \theta)$,

$\int_{-\Lambda}^{\Lambda} d z \int_{0}^{2 \pi} d \theta \tilde{r}_{i}=0$

It is easy to check that the set of equations (9) admits a solution of the form $\tilde{r}_{i}(z, \theta)=f_{i}(z) \cos \left(\theta-\delta_{i 1} \alpha\right)$ and $\tilde{p}_{i}=0$, the functions $f_{i}(z)$ verifying the conditions
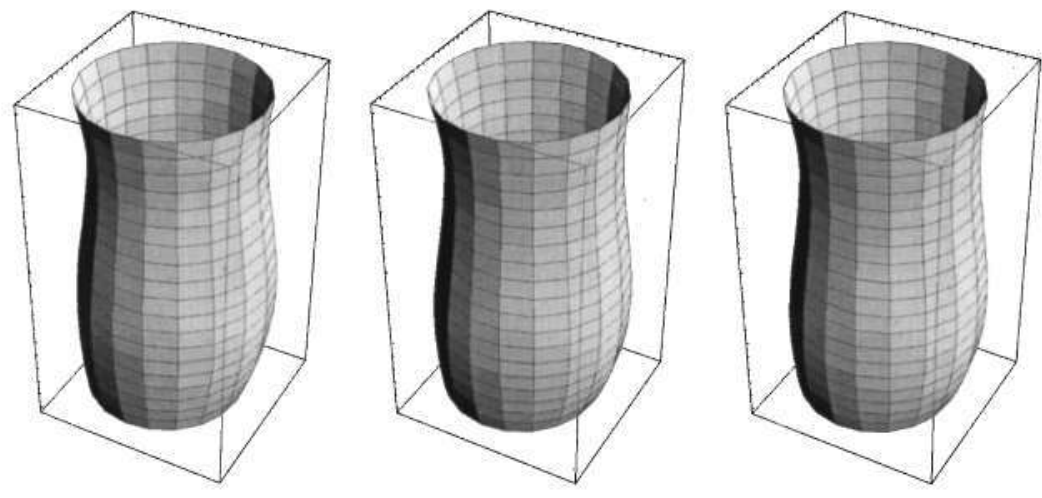

FIG. 5. Liquid bridge equilibrium shape obtained from the analytical expansion (left-hand side), the finite difference scheme (center) and the approximate numerical method (right-hand side) for $V=1.1, \Lambda=2, H=0.05$, $B_{\alpha}=0.15, W=0, B_{l}=0.05, e=0.05, \alpha=0$. 

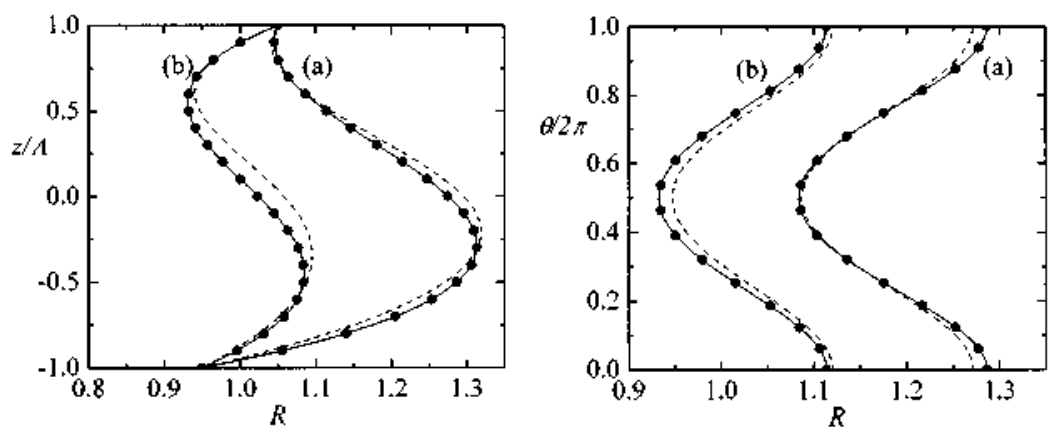

FIG. 6. Plot of the equilibrium contour for $V=1.2, \Lambda$ =2. $H=0.05, B_{0}=0.15, W=0, B_{t}=0.05$, and $e=\alpha$ $=0$. The circles represent the finite difference scheme results, whereas the solid lines and the dashed lines correspond to the solutions (8a) and (3a), respectively. Figure shows the intersection between the liquid bridge interface and the planes $\theta=0$ (a) and $\theta=\pi$ (b) (lefthand graph), and $z=-\Lambda / 2$ (a) $z=\Lambda / 2$ (b) (right-hand graph).

$$
\begin{aligned}
& W \widetilde{R}^{2}\left(1+\widetilde{R}_{z}^{2}\right)^{1 / 2} f_{l}+\frac{\widetilde{R}_{z}\left(\mathbf{1}+\widetilde{R}_{z}^{2}-3 \widetilde{R} \widetilde{R}_{z z}\right)}{\left(1+\widetilde{R}_{z}^{2}\right)^{2}} f_{i_{z}}+\frac{\widetilde{R}}{\mathbf{1}+\widetilde{R}_{z}^{2}} f_{i_{z z}} \\
& \quad+\delta_{i 1} \tilde{R}^{2}\left(1+\widetilde{R}_{z}^{2}\right)^{1 / 2}=0,
\end{aligned}
$$$$
f_{i}( \pm \Lambda)= \pm \delta_{i 2}
$$

Once the axisymmetric contour $\tilde{R}(z)$ is known, $(10)$ constitutes an elemental two-point boundary-value problem which is solved using again the shooting method. For the particular case of $Y=1$ and $H=B_{a}=W=0, \widetilde{R}=1$ and $\widetilde{P}=1$ whatever the slenderness $\Lambda$ is. In this case, $f_{i}(z)$ coincides with the corresponding function obtained in Sec. III and given in the Appendix: $f_{1}(z)=\left(\Lambda^{2}-z^{2}\right) / 2$ and $f_{2}(z)=z / \Lambda$. In Fig. 4 we plot $f_{i}(z)$ for $\mathrm{V}^{\prime}=1.1, \Lambda=2, H=0.05, B_{a}=0.15$ and $W^{\prime}=0$ along with the above analytical functions. As can be observed, the differences are negligible for $f_{2}(z)$ but noticeable for $f_{1}(z)$

The numerical approach described in this subsection deals with the problem (I) by transforming it to the two coupled boundary-value problems (7) and (10). First. given the values of $V, \Lambda, H, B_{a}$ and $W$, the axisymmetric contour $\widetilde{R}(z)$ is obtained numerically from (7). Second. the functions $f_{i}(z)$ are also calculated numerically by integrating $(10)$. The solution ( 8 ) can be considered as "exact" for $B_{l}=e=0$, and is expected to be quite accurate for small values of these parameters. In addition. there is no restriction on the values of the parameters characterizing the problem (in particular. the value of $\alpha$ ). Another Mathematica package programmed by the authors, directly provides the numerical value of the function $R\left(V, \Lambda, H, B_{a}, W, B_{l}, e, \alpha ; z, \theta\right)$ without other specifications.

\section{THEORETICAL RESULTS}

In order to test the validity of the three theoretical approaches used in this work, we have compared the results obtained from them. Figure 5 shows the liquid bridge contour computed using the analytical expansion (left-hand side), the finite difference scheme (center), and the approximate numerical method described in the previous section (right-hand side). The choice for the parameters characterizing the problem was $\left\{V^{\prime}=1.1 . \Lambda=2 . H=0.05, B_{a}=0.15 . W\right.$ $\left.=0 . B_{t}=0.05, e=0.05, \alpha=0\right\}$. Despite the fact that both axisymmetric and nonaxisymmetric perturbations are strong, the agreement is quite good. Nevertheless. small differences can be observed between the contour obtained from the analytical expansion and those shapes calculated numerically. Figures 6 and 7 show the intersection between the liquid bridge interface and the planes $\theta=0, \theta=\pi, z=-\Lambda / 2$ and $z=\Lambda / 2$ as obtained from the three theoretical approaches used in this work. The liquid bridges considered are also subjected to strong axisymmetric and nonaxisymmetric perturbations, so the deviations from cylindrical are noticeable (the maximum deviation observed in Figs. 6 and 7 are around $30 \%$ and $15 \%$, respectively). Let us now consider the finite difference scheme results as a reference against which to check the other theoretical predictions. As can be observed in Figs. 6 and 7 , the solution (8a) significantly improves the analytical results. In fact. the agreement between the results of the finite difference scheme and of the solution (8a) is excellent in both cases.

In order to perform a more exhaustive test, we calculated the relative error $E(z, \theta)$ defined as

$$
E(z, \theta)=\frac{\left|R(z, \theta)-R_{\mathrm{FD}}(z, \theta)\right|}{\left|\mathrm{I}-R_{\mathrm{FD}}(z, \theta)\right|} .
$$
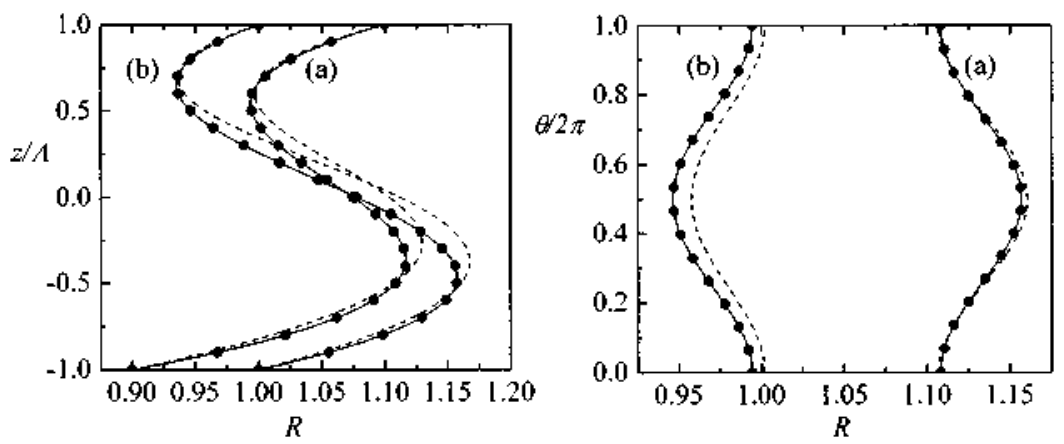

FIG. 7. The same as in Fig. 6 but for $V=1.1, \Lambda=2$, $H=0.05, B_{a}=0.15, W=0, B_{t}=0, e=0.05$, and $\alpha=0$. 

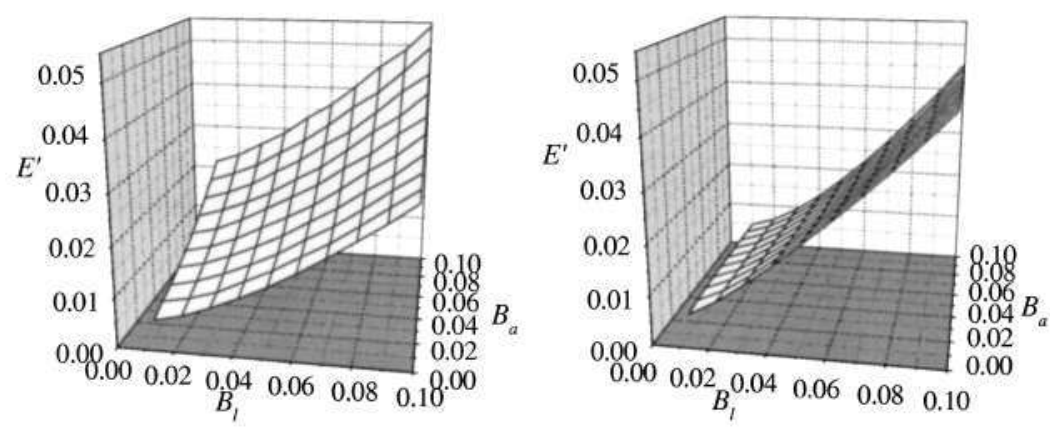

FIG. 8. Relative error $E^{\prime}$ as a function of $B_{a}$ and $B_{l}$ for $V=1, \Lambda=2, H=e=W=\alpha=0$. In the left-hand graph $R(z, \theta)$ in Eq. (11) was calculated using the solution (3a), while in the right-hand graph $R(z, \theta)$ was obtained from $(8 a)$.

where $R(z, \theta)$ represents the equilibrium contour as obtained from either the analytical expansion (3a) or the numerical solution ( $8 \mathrm{a})$, and $R_{\mathrm{FD}}(z, \theta)$ is the liquid bridge contour calculated by means of the finite difference scheme. In principle, one must expect the value of $E(z, \theta)$ to increase as the distance $\left|1-R_{\mathrm{FD}}(z, \theta)\right|$ increases. Therfore, $E^{\prime} \equiv E\left(z^{\prime}, \theta^{\prime}\right)$ can be considered as an upper bound of the relative error $E(z, \theta)$. Here, the coordinates $z^{\prime}$ and $\theta^{\prime}$ correspond to the surface element for which $\left|1-R_{\mathrm{FD}}(z, \theta)\right|$ is maximum. Figure 8 shows the dependence of $E^{\prime}$ on both the axial and lateral Bond numbers. The rest of the parameters characterizing the liquid bridge were fixed. In the left-hand graph $R(z, \theta)$ in Eq. (11) was calculated using the expansion (3a), while in the right-hand graph $R(z, \theta)$ was obtained using the solution (8a). At a qualitative level, the agreement between the analytical and the finite difference method results was satisfactory even for large values of the forces acting on the liquid bridge. For instance, for $B_{a}=B_{l}=0.1, \mid R\left(z^{\prime}, \theta^{\prime}\right)$ $-R_{\mathrm{FD}}\left(z^{\prime}, \theta^{\prime}\right) \mid=0.0143512$, while the maximum deviation of the bridge contour from a cylinder is $\left|1-R_{\mathrm{FD}}\left(z^{\prime}, \theta^{\prime}\right)\right|$ $=0.264515$. Therefore, in spite of the clear deviation of the liquid bridge contour from cylindrical, the analytical expansion provides moderately accurate predictions. Nevertheless, the errors associated with the expansion (3) increase monotonically as the perturbation parameters $B_{a}$ and $B_{l}$ increase. This behavior is even more apparent for larger values of the slenderness $\Lambda$.

The agreement between the results obtained from the two numerical methods is excellent for small values of the lateral Bond number $B_{l}$, whatever the value of the axial Bond number $B_{a}$ is. For instance, for $B_{a}=0.1$ and $B_{l}=0.01,\left|R\left(z^{\prime}, \theta^{\prime}\right)-R_{\mathrm{FD}}\left(z^{\prime}, \theta^{\prime}\right)\right|=5.11405 \times 10^{-4}$, while $\left|1-R_{\mathrm{FD}}\left(z^{\prime}, \theta^{\prime}\right)\right|=0.105010$. Because the numerical solution (8a) is "exact" for the axisymmetric case $B_{l}=0$, the excellent agreement observed in this limit can be considered as a validation of the finite difference scheme. As must be expected, the errors associated with the expansion (8a) increase monotonically as $B_{l}$ increases, but remain constant as $B_{a}$ increases. Finally, it must be pointed out that for large values of the perturbation parameter $B_{l}$, the first-order expansion (8a) provides results of similar accuracy to those obtained from the second-order expansion (3a).

\section{EXPERIMENTS}

In order to perform the experiments described in the following, the experimental arrangement sketched in Fig. 9 was used. The apparatus consists of a liquid bridge cell (A) and a video camera $(B)$, both mounted on a platform $(C)$. The platform in turn is mounted on a tilting table (D), and can be rotated at angle $\theta$ around a fixed point of the tilting table. The tilting table is articulated along an axis to a fixed horizontal base (E), with $\phi$ being the angle between the tilting table and the fixed based. An illumination system (F) is also used.

Experiments were performed using the so-called neutral buoyancy or plateau technique. ${ }^{6}$ The liquid column is formed inside another liquid (a surrounding bath) of nearly the same density but immiscible with the former. Dimethyl-silicone oil is used as the working liquid, and the surrounding bath is a mixture of methanol and distilled water. The values of the interfacial tension and of the surrounding bath density can be modified by adjusting the ratio of alcohol to water of the mixture. All experiments were performed at a temperature of $20 \pm 1^{\circ} \mathrm{C}$. The liquid bridge cell (LBC) consists of a chamber inside which the liquid bridge is formed. Because the chamber is air-tight, no alcohol evaporation occurs, so that the surrounding bath mass is constant. The LBC chamber is a $0.04 \mathrm{~m}$ sided aluminum cube, with two opposing faces made of a transparent plastic. The liquid bridge is formed between two equal disks $(H=0) 0.01 \mathrm{~m}$ in diameter. One of the disks is connected to the piston of the syringe and can be displaced along its axis by using a micrometer screw. The other disk is fixed to the opposite side of the test chamber such that both disks remain in coaxial alignment $(e=0)$. Liquid can be injected and removed from the liquid bridge through a hole in the center of the moving disk which connects it with the syringe. Two quick-disconnect valves are used to fill the test chamber with the surrounding liquid.

In each experiment the sequence is as follows. First, the moving disk is axially displaced until it comes very close to

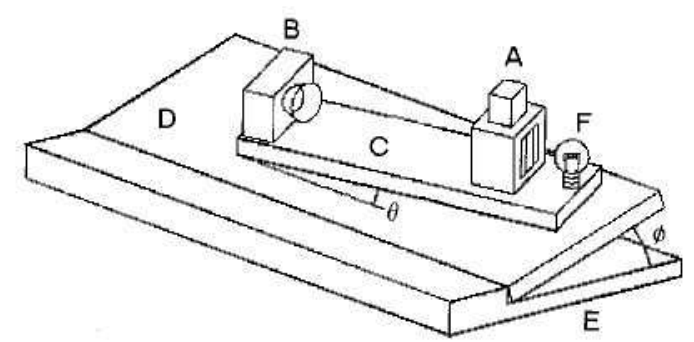

FIG. 9. Sketch of the experimental apparatus: (A) liquid bridge cell, (B) video camera, (C) rotating platform, (D) tilting table, (E) fixed horizontal base, and (F) illumination system. 


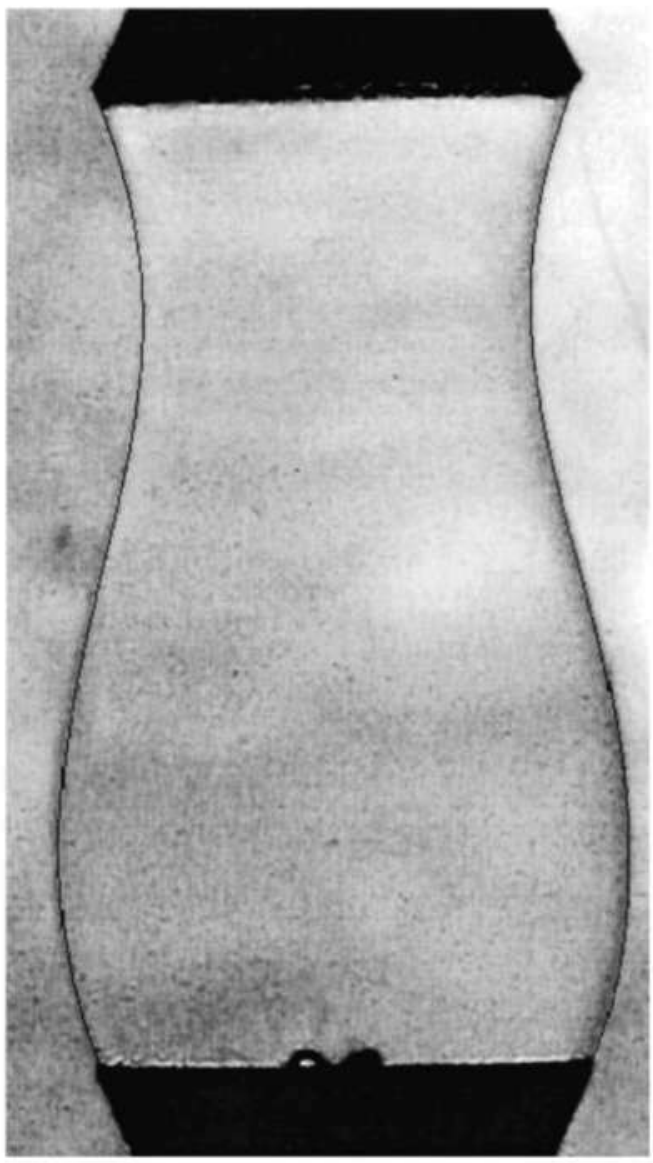

FIG. 10. Liquid bridge contour for $V=0.99, \Lambda=2.0, B_{a}=0.19$, and $B_{l}$ $=0.0$. The solid lines represent the contour obtained by fitting the axisymmetric numerical solution of the capillary equation.

the lower one. A small bridge which fills the gap between both disks is formed. The LBC chamber, set upright, is filled with the surrounding bath of the desired density. The disk connected to the piston of the syringe is displaced along its axis until the desired slenderness is reached. The amount of liquid injected during this process causes the dimensionless volume $V$ of the liquid bridge to be $V=1 \pm 0.01$. To modify this value, liquid can be either injected $(V>1)$ or removed $(V<1)$ from the liquid bridge. At this stage, the values of the volume $V$ and of the interfacial tension $\sigma$ are calculated by fitting the solution of the capillary equation to the contour of the liquid bridge formed inside the LBC chamber. Since the LBC chamber is upright, the liquid bridge is still axisymmetric, so that the numerical solution $\widetilde{R}(z)$ of the capillary equation is assumed to be known. ${ }^{8}$ Once the values of $V$ and $\sigma$ have been calculated, the LBC is mounted on the platform and the angles $\theta$ and $\phi$ are fixed. In this way, we obtain nonzero components of the gravity along the axial and lateral directions. For each orientation, the camera records the liquid bridge contour. The sequence is repeated varying the experimental conditions.

For the sake of illustration, Fig. 10 shows an axisymmetric liquid bridge contour formed inside the LBC chamber. Once the value of the slenderness $\Lambda$ is fixed, the deformation of the liquid bridge interface only depends on the reduced
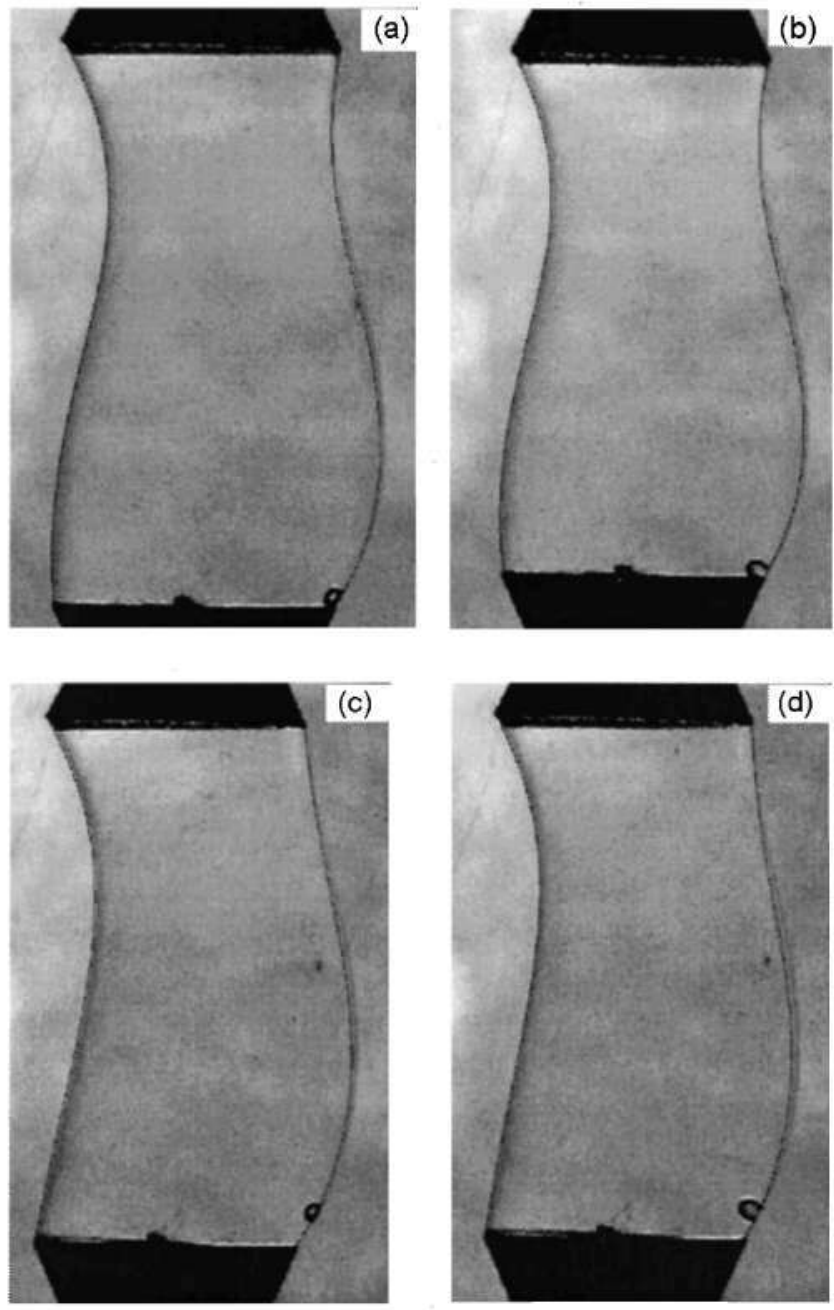

FIG. 11. Liquid bridge contour for $V=0.99$ and $\Lambda=2.0$. The photographs (a) and (b) correspond to $B_{a}=0.17$ and $B_{l}=0.097$, whereas (c) and (d) corresponds to $B_{a}=0.097$ and $B_{1}=0.17$. In addition, $\theta=0^{\circ}, 180^{\circ}$ for (a) and (c), with $\theta=30^{\circ}, 210^{\circ}$ for (b) and (d) (the first and the second values of $\theta$ correspond to the right-hand and left-hand contours, respectively). The solid lines are the predictions obtained from the finite difference scheme.

volume $V$ and the axial Bond number $B_{a}$. As mentioned before, the values of $V$ and $B_{a}$ are inferred by fitting the numerical solution $\widetilde{R}(z)$ of the capillary equation to the axisymmetric liquid bridge contour. The value of the interfacial tension is then obtained: $\sigma=\Delta \rho g R_{0}^{2} / B_{a}$. The solid lines plotted in Fig. 10 correspond to the numerical solution for $V=0.99, \Lambda=2.0$, and $B_{a}=0.19$. As can be observed, there is excellent agreement between the liquid bridge contour and the numerical solution for those values of $V$ and $B_{a}$. Once the interfacial tension has been calculated, the values of both the axial and the lateral Bond numbers are set by mounting the $\mathrm{LBC}$ on the platform and fixing the angle $\phi$ :

$$
B_{a}=\frac{\cos \phi \Delta \rho g R_{0}^{2}}{\sigma}, \quad B_{l}=\frac{\sin \phi \Delta \rho g R_{0}^{2}}{\sigma} .
$$

Figure 11 shows the liquid bridge equilibrium contours for $V=0.99$ and $\Lambda=2.0$ subjected to both axial and lateral forces. The photographs (a) and (b) correspond to $B_{a}=0.17$ and $B_{l}=0.097$, and (c) and (d) correspond to $B_{a}=0.097$ and 

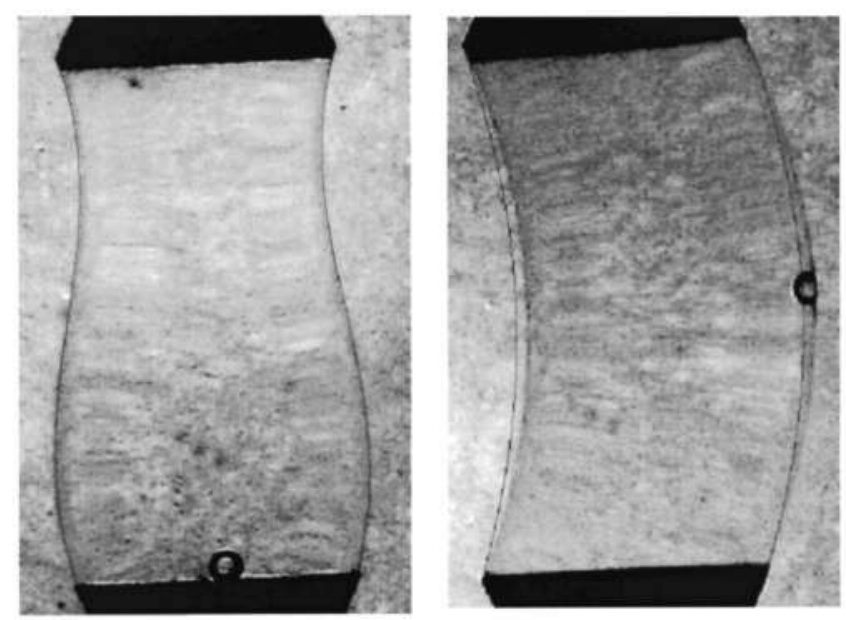

FIG. 12. Liquid bridge contour for $V=1.0$ and $\Lambda=2.0$. The photographs correspond to $B_{a}=0.18$ and $B_{l}=0.0$ (left-hand photograph), and to $B_{a}$ $=0.0$ and $B_{l}=0.18$ (right-hand photograph). The solid lines are the predictions obtained from the finite difference scheme. In the nonaxisymmetric case, $\theta=0^{\circ}$ and $180^{\circ}$ for the right-hand and the left-hand contours, respectively.

$B_{l}=0.17$. They show the liquid bridge interface shape for different values of $\theta$. The solid lines are the theoretical predictions obtained from the finite difference scheme. Qualitatively, the agreement between the theoretical contours and the experimental ones is good, despite the noticeable deviation of the liquid bridge shape from the cylinder. The differences observed increase as the value of the lateral Bond number increases. This behavior is more apparent in the experiment shown in Fig. 12. The photographs show the liquid bridge contour for $V=1.0, \Lambda=2.0, B_{a}=0.0$, and $B_{l}=0.18$ for the nonaxisymmetric case. Now the agreement between the theoretical shape and the experiment is worse. In principle, there is no reason to expect the results obtained from the finite difference scheme to get worse as $B_{l}$ increases. Comparison between contours calculated from the different
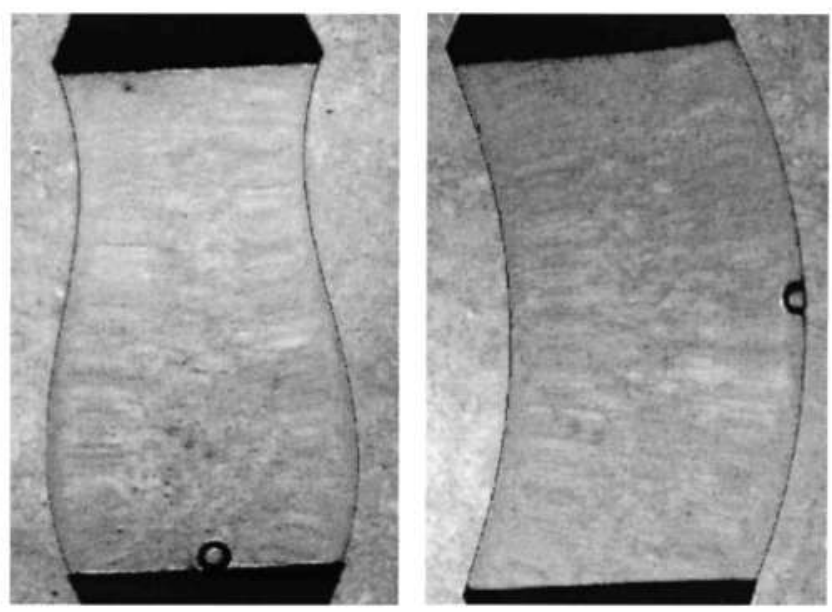

FIG. 13. The same as in Fig. 12 but the theoretical contours are calculated for $B_{a}=0.22$ and $B_{l}=0.0$ (left-hand photograph), and $B_{a}=0.0$ and $B_{l}$ $=0.22$ (right-hand photograph).
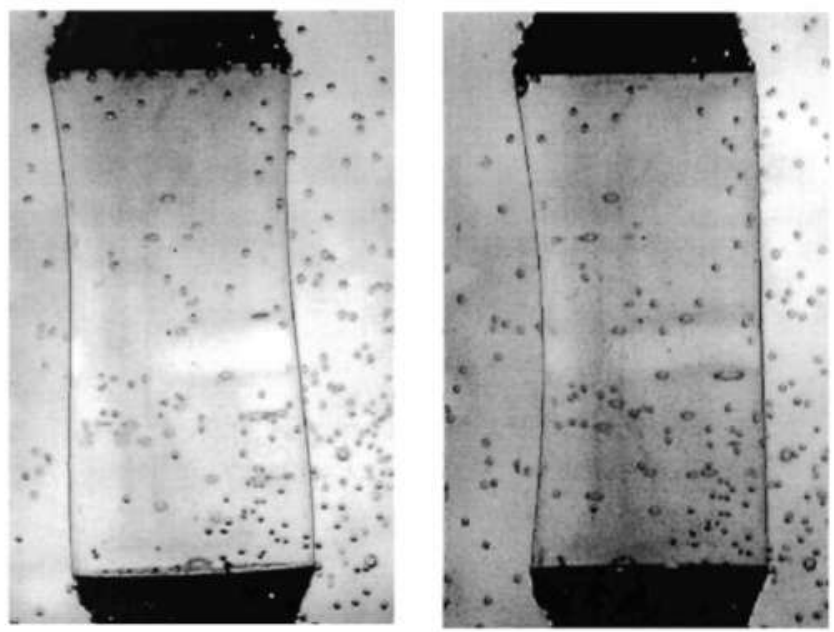

FIG. 14. Liquid bridge and theoretical contours for $V=0.897, \Lambda=2.0$, and $B_{a}=0.050, B_{l}=0.0$ (left-hand photograph), and $B_{a}=0.0, B_{l}=0.050$ (righthand photograph). The solid lines are the predictions obtained from the finite difference scheme. In the nonaxisymmetric case, $\theta=0^{\circ}$ and $180^{\circ}$ for the right-hand and the left-hand contours, respectively.

theoretical approaches described in this work supports the above statement. Therefore, the deviations observed could be caused by experimental factors.

When the Bond number is sufficiently large in value, the axisymmetric liquid bridge shape becomes practically insensitive to small variations of this parameter. This implies that slight errors associated with either experimental conditions or the image post-processing produce relevant discrepancies in the calculation of the Bond number. In Fig. 13 we show the same liquid bridge considered in Figs. 12, and the numerical results obtained for $B_{a}=0.22, B_{l}=0.0$ (left-hand photograph), and $B_{a}=0.0$ and $B_{l}=0.22$ (right-hand photograph). In this case the value of the surface tension was calculated by fitting the theoretical prediction of the finite difference method to the nonaxisymmetric contour shown in the right-hand photograph. As can be observed, the agree-
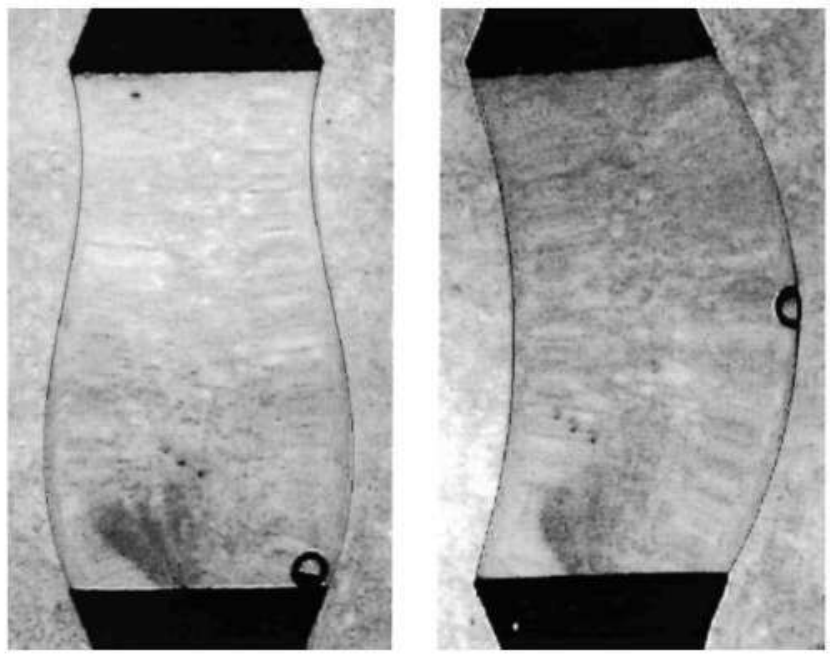

FIG. 15. The same as in Fig. 14 but for $V=1.14, \Lambda=1.9$, and $B_{a}=0.23$, $B_{l}=0.0$ (left-hand photograph), and $B_{a}=0.0, B_{l}=0.23$ (right-hand photograph). 
ment between the numerical solution and the experiment is excellent in both the axisymmetric and nonaxisymmetric cases. Similar results were obtained for other liquid bridges considered (see, for instance, the experiments shown in Figs. 14 and 15 ).

In most previous experiments with liquid bridges, the surface tension was measured following basically the same procedure used in this present work (see, for instance. Refs. $6,7.15$, and 16). From the results described above. one could speculate that those measurements would be significantly improved (at less for large values of the Bond number) if nonaxisymmetric experimental contours were fitted to those calculated from the finite difference scheme.

\section{CONCLUDING REMARKS}

The calculation of the equilibrium liquid bridge contour is a hard task because of the large number of parameters involved and the complicated mathematical structure of the capillary equation. Indeed, the local mean curvature of the surface is a nonlinear function of the liquid bridge shape $R(z, \theta)$ and of its partial derivatives. Therefore, the search for exact analytical solutions is impractical [except the trivial solution $R(z, \theta)=P=1$ ]. We have here described an analytical and numerical study of the equilibrium interface shape of a liquid bridge subjected to both axisymmetric and nonaxisymmetric perturbations. The analytical approach used an expansion around the cylindrical solution. The numerical data were computed from a finite difference scheme and from an approximate numerical method. From these calculations, one does not known a priori whether the liquid bridge is stable under the conditions considered. It must be assumed however that, at least for configurations close to cylindrical. liquid bridges are stable.

In principle, the convergence of the finite difference scheme is not guaranteed. First, we have shown that the discretization mesh size does not play a relevant role in our calculations. Second, once the parameters characterizing the liquid bridge are fixed, the Newton-Raphson iteration converges to a unique solution independently of the initial guess used in the iteration. Therefore, the finite difference scheme used to calculate the liquid bridge contour can be considered as consistent and stable. Nevertheless, because we are dealing with a nonlinear problem. consistency and stability do not necessarily imply convergence. ${ }^{17}$ In any case, the Newton-Raphson method always leads to a solution in very good agreement with that obtained from the other numerical approach presented in this paper. Therefore, one could expect that the finite difference scheme provides accurate predictions even for large perturbations. These predictions can be considered as a reference against which to check other theoretical approaches. The only restriction is that the plane containing the axes of the disks must also contain the lateral force direction $(\alpha=0)$. From the comparison between analytical predictions and numerical data, we also concluded that the validity of the former can be extended to liquid bridges showing moderate deviations from the cylindrical configuration.
The comparison between equilibrium contours obtained from the finite difference method and from experiment confirms that this method yields accurate predictions even for large deviations from cylindrical. Nevertheless, significant discrepancies can be observed for strong nonaxisymmetric perturbations. In this extreme case the discrepancy is presumably due to an error in measuring the surface tension from the axisymmetric contour. In fact. one can find excellent agreement by adjusting the value of this latter quantity from the nonaxisymmetric shape. This result suggests a new way to measure the surface tension in experiments with liquid bridges.

\section{ACKNOWLEDGMENTS}

This research was supported by the Ministerio de Educación y Cultura and by the Junta de Extemadura (Fondo Social Europeo) through Grant No. 1FD970-1669.

\section{APPENDIX: SOLUTION TO THE ANALYTICAL EXPANSION}

The functions $r_{i}$ corresponding to the order $\epsilon_{i}$ of the expansion (3a) are the following:

$$
\begin{aligned}
& r_{1}=z-\Lambda \frac{\sin z}{\sin \Lambda}, \\
& r_{2}=\frac{1}{2} N(\cos z-\cos \Lambda), \quad N=\frac{\Lambda}{\sin \Lambda-\Lambda \cos \Lambda}, \\
& r_{3}=\frac{\sin z}{\sin \Lambda}, \\
& r_{4}=0 \\
& r_{5}=\frac{z}{\Lambda} \cos \theta . \\
& r_{6}=\frac{1}{2}\left(\Lambda^{2}-z^{2}\right) \cos (\theta-\alpha) .
\end{aligned}
$$

The functions $r_{i j}$ corresponding to the order $\epsilon_{i} \epsilon_{j}$ of the expansion (3a) are the following:

$$
\begin{aligned}
r_{11}= & T_{11}(z)-T_{11}(\Lambda), \\
T_{11}(z)= & \frac{A}{2 \sin \Lambda}\left(z^{2}-\Lambda^{2}+4\right) \cos z-\frac{\Lambda^{2} \sin ^{2} z}{2 \sin ^{2} \Lambda}+z^{2}, \\
r_{12}=r_{21}= & T_{12}(z)-T_{12}(\Lambda) \frac{\sin z}{\sin \Lambda}, \\
T_{12}(z)= & \frac{1}{4} N\left(\frac{1}{2} z^{2} \sin z-2 z \cos \Lambda+\frac{\Lambda}{\sin \Lambda} \cos z \sin z\right. \\
& \left.-\frac{\Lambda \cos \Lambda}{\sin \Lambda} z \cos z\right), \\
r_{13}= & r_{31}=
\end{aligned}
$$




$$
\begin{aligned}
T_{13}(z)= & \frac{1}{4 \sin \Lambda}\left[\left(4-\Lambda^{2}+z^{2}-2 N \Lambda \sin \Lambda\right) \cos z\right. \\
& \left.+\frac{2 \Lambda}{\sin \Lambda} \cos ^{2} z\right] \\
r_{14}=r_{41}= & \frac{\Lambda \sin z}{4 \sin ^{2} \Lambda}(\Lambda \cos \Lambda+2 \sin \Lambda) \\
& -\frac{1}{2} \Lambda \sin z(2 \sin \Lambda+\Lambda \cos z) \\
r_{15}=r_{51}= & {\left[T_{15}(z)-T_{15}(\Lambda)\right] \cos \theta } \\
T_{15}(z)= & -\frac{1}{2} \Lambda\left(\frac{1}{2} z^{2}+\frac{\Lambda}{\sin \Lambda} \cos z\right) \\
r_{16}=r_{61}= & {\left[T_{16}(z)-T_{16}(\Lambda) \frac{z}{\Lambda}\right] \cos (\theta-\alpha) . } \\
T_{16}(z)= & \frac{1}{2} \frac{\Lambda}{\sin \Lambda}(z \cos z-3 \sin z) . \\
r_{22}=T_{22}(z)-T_{22}(\Lambda) . & \\
T_{22}(z)= & \frac{1}{4} N^{2}\left\{\frac{1}{2} \sin { }^{2} z\right. \\
& +\cos \Lambda[(2-N \Lambda \sin \Lambda) \cos z-z \sin z]\} .
\end{aligned}
$$$$
r_{23}=r_{32}=T_{23}(z)-T_{23}(\Lambda) \frac{\sin z}{\sin \Lambda} .
$$$$
T_{23}(z)=\frac{N \cos z}{4 \sin \Lambda}(z \cos \Lambda-\sin z) .
$$$$
r_{24}=r_{42}=\frac{1}{8} N[(I-N \Lambda \sin \Lambda) \cos z-z \sin z
$$$$
\left.-\frac{N}{\Lambda} \sin \Lambda \cos \Lambda+N\right] \text {, }
$$$$
r_{25}=r_{52}=\frac{N}{4 \Lambda}\left(\frac{z}{\Lambda} \sin \Lambda-\sin z\right) \cos \theta .
$$$$
r_{26}=r_{62}=\left[T_{26}(z)-T_{26}(\Lambda)\right] \cos (\theta-\alpha) \text {, }
$$$$
T_{26}(z)=\frac{1}{4} N\left(z \sin z+3 \cos z+\frac{1}{2} z^{2} \cos \Lambda\right) \text {. }
$$$$
r_{33}=T_{33}(z)-T_{33}(\Lambda) \text {. }
$$$$
T_{33}(z)=\frac{\cos ^{2} z}{2 \sin ^{2} \Lambda}-\frac{1}{2} N \cos z,
$$$$
r_{34}=r_{43}=\frac{1}{8 \sin ^{2} \Lambda}(z \sin \Lambda \cos z-\Lambda \cos \Lambda \sin z) \text {. }
$$$$
r_{35}=r_{53}=\frac{1}{2 \Lambda \sin \Lambda}(\cos z-\cos \Lambda) \cos \theta .
$$$$
r_{36}=r_{63}=-\frac{1}{\Lambda} r_{16}
$$$$
r_{44}=0
$$$$
r_{45}=r_{54}=\frac{1}{6 \Lambda} z\left(\Lambda^{2}-z^{2}\right) \cos \theta,
$$

$$
\begin{aligned}
& r_{46}=r_{64}=\frac{1}{24}\left(z^{4}-6 \Lambda^{2} z^{2}+5 \Lambda^{4}\right) \cos (\theta-\alpha), \\
& r_{55}=\frac{1}{4 \Lambda^{2}}\left[\left(z^{2}+1-\frac{\cosh (\sqrt{3} z)}{\cosh (\sqrt{3} \Lambda)}\right) \cos (2 \theta)-z^{2}\right], \quad \text { (A334) } \\
& r_{56}=r_{65}=\frac{1}{8 \Lambda}\left[\left(\Lambda^{2}-2\right) z-z^{3}+2 \Lambda \frac{\sinh (\sqrt{3} z)}{\sinh (\sqrt{3} \Lambda)}\right] \cos (2 \theta \\
& \quad-\alpha)+T_{56}(z)-T_{56}(\Lambda) \frac{\sin z}{\sin \Lambda}, \\
& T_{56}(z)=\frac{\cos \alpha}{8 \Lambda}\left[z^{3}-\left(\Lambda^{2}+6\right) z\right] . \\
& r_{66}=\frac{1}{16}\left[\left(z^{2}-\Lambda^{2}\right)^{2}+4 z^{2}+\frac{8}{3}-\left(4 \Lambda^{2}+\frac{8}{3}\right) \frac{\cosh (\sqrt{3} z)}{\cosh (\sqrt{3} \Lambda)}\right] \\
& \quad \times \cos [2(\theta-\alpha)]+T_{66}(z)-T_{66}(\Lambda), \\
& T_{66}(z)=\frac{1}{2} N \Lambda^{2} \cos z-\frac{1}{8} z^{2}\left(\frac{1}{2} z^{2}-\Lambda^{2}-6\right) .
\end{aligned}
$$

The parameters $p_{i}$ corresponding to the order $\epsilon_{i}$ are

$$
p_{1}=p_{3}=p_{5}=p_{6}=0, \quad p_{2}=\frac{1}{2} N \cos \Lambda, \quad p_{4}=-\frac{1}{2} \text {. }
$$

Finally, the values of $p_{i j}$ corresponding to the order $\epsilon_{i} \epsilon_{j}$ are equal to zero except

$$
\begin{aligned}
p_{11}= & 2 \Lambda \cot \Lambda+\frac{\Lambda^{2}}{2 \sin ^{2} \Lambda}+\frac{1}{2}\left(\Lambda^{2}-5\right), \\
p_{13}= & p_{31}=\frac{1}{2} \Lambda \cot ^{2} \Lambda+\cos \Lambda\left(\frac{1}{\sin \Lambda}-\frac{1}{2} N \Lambda\right), \\
p_{22}= & -\frac{1}{8} N^{2}\left[\cos ^{2} \Lambda(2 \Lambda N \sin \Lambda-5)\right. \\
& +2 \Lambda \sin \Lambda \cos \Lambda-1] . \\
p_{24}= & p_{42}=\frac{1}{8} N^{2}-\cos \Lambda\left(\frac{N^{2} \sin \Lambda}{8 \Lambda}+\frac{1}{4} N\right), \\
p_{33}= & \frac{1}{2} N \cos \Lambda-\frac{1}{2} \cot { }^{2} \Lambda, \\
p_{55}= & -\frac{1}{4 \Lambda^{2}}, \\
p_{66}= & \frac{1}{4} N\left[\left(\Lambda^{2}+6\right) \cos \Lambda+(\Lambda-6 / \Lambda) \sin \Lambda\right] .
\end{aligned}
$$

${ }^{1}$ J. Meseguer, J. M. Perales. I. Martinez, N. A. Bezdenejnylh, and A. Sanz, "Hydrostatic instabilities in floating zone crystal growth process." Crystal Growth Research 5, 27 (1999).

${ }^{2}$ E. Bayramli, A. Abou-Obeid, and T. G. M. van de Ven, "Liquid bridges between spheres in a gravitational field," J. Colloid Interface Sci. 116, 351 (1987).

${ }^{3} \mathrm{~A}$. Leverón-Simavilla and J. M. Perales, "Equilibrium shapes of nonaxisymmetric liquid bridges of arbitrary volume in gravitational fields and their potential energy," Phys. Fluids 7. 1204 (1995).

${ }^{4}$ A. Laveron-Simavilla and E. Checa, "Effect of a lateral gravitational field on the nonaxisymmetric equilibrium shapes of liquid bridges held between eccentric disks and of volumes equal to those of cylinders," Phys. Fluids 9. 817 (1997).

${ }_{5}^{5}$ J. Meseguer. L. A. Slobozhanin, and J. M. Perales, "A review on the stability of liquid bridges," Adv, Space Res, 16, 5 (1995).

${ }^{6} \mathrm{~F}$. Zayas, J. I. D. Alexander, J. Meseguer, and J. F. Ramus, "On the sta- 
bility limits of long nonaxisymmetric cylindrical liquid bridges," Phys. Fluids 12, 979 (2000).

${ }^{7}$ J. Meseguer, N. A. Bezdenejnykh, and P. Rodriguez de Francisco, "On the use of liquid bridges as accelerometers," Microgravity Sci. Technol. 9, 1 (1996).

${ }^{8}$ J. M. Montanero, G. Cabezas, J. Acero, and F. Zayas, "Using rotating liguid bridges as accelerometers," Microgravity Sci. Technol. (to be published).

${ }^{9} \mathrm{~V}$. M. Shevtsova and J. C. Legros, "Oscillatory convective motion in deformed liquid bridges," Phys. Fluids 10, 1621 (1998).

${ }^{10}$ L. B. Summer, G. P. Neitzel, J. P. Fontaine, and P. Dell'Aversana "Oscillatory thermocapillary convection in liquid bridges with highly deformed free surfaces: experiments and energy-stability analysis," Phys. Fluids 13. 107 (2001).

${ }^{11} \mathrm{~K}$. Brakke. "The surface evolver," Akust. Z. Experimental Math. 1, 141 (1992).
12J. M. Perales, A. Sanz, and D. Rivas, "Eccentric rotation of a liquid bridge." Appl. Microgravity Tech. II 4. 193 (1990).

${ }^{13} \mathrm{H}$. P. Kruse, "Bifurcation of rotating inviscid licuid bridges with fixed contact line," Z. Angew. Math. Mech. 80. 411 (2000).

${ }^{14}$ W. H. Press, B. P. Flannery, S. A. Teukolsky, and W. T. Vetterling, $N u$ mencal Recipes in Fortran 77: The Art of Scientific Computmg (Cambridge University Press, Cambridge, 1986).

${ }^{15}$ J. Meseguer, L. A. Mayo, J. C. Llorente, and A. Fernández, "Experiments with licjuid bridges in simulated nicrogravity," J. Cryst. Growth 73, 609 (1985).

${ }^{16}$ A. Ramos, H. González, and A. Casatellanos, "Experiments on dielectric liquid bridges subjected to axial electric fields," Phys. Fluids 6, 3206 (1994).

${ }^{17} \mathrm{P}$. J. Roache, Fundamentals of Computational Fiud Dynamics (Hermosa, Albuquerque, NM, 1998). 